\title{
Role of external and internal perturbations on ferromagnetic phase transitions in manganites: Existence of tricritical points
}

\author{
Prabir K. Mukherjee, ${ }^{1}$ Prosenjit Sarkar, ${ }^{2}$ and Amit K Chattopadhyay ${ }^{3}$ \\ ${ }^{1}$ Department of Physics, Government College of Engineering and Textile Technology, \\ 12 William Carey Road, Serampore, Hooghly-712201, India* \\ ${ }^{2}$ Department of Physics, Serampore College, Serampore 712201, India ${ }^{\dagger}$ \\ ${ }^{3}$ Aston University, Mathematics, System Analytics Research Institute, Birmingham B4 7ET, UK
}

(Dated: June 7, 2018)

\begin{abstract}
A phenomenological mean-field theory is presented to describe the role of external magnetic field, pressure and chemical substitution on the nature of ferromagnetic (FM) to paramagnetic (PM) phase transition in manganites. The application of external field (or pressure) shifts the transition, leading to a field (or pressure) dependent phase boundary along which a tricritical point is shown to exist where a first-order FM-PM transition becomes secondorder. We show that the effect of chemical substitution on the FM transition is analogous to that of external perturbations (magnetic field and pressure); this includes the existence of a tricritical point at which the order of transition changes. Our theoretical predictions satisfactorily explain the nature of FM-PM transition, observed in several systems. The modeling hypothesis has been critically verified from our experimental data from a wide range of colossal magnetoresistive manganite single crystals like $\mathrm{Sm}_{0.52} \mathrm{Sr}_{0.48} \mathrm{MnO}_{3}$. The theoretical model prediction of a tricritical point has been validated in this experiment which provides a major ramification of the strength of the model proposed.
\end{abstract}

PACS numbers: 75.20.-g,75.30.Kz,75.40.Cx 


\section{INTRODUCTION}

The physics of critical phenomena and phase transitions is often a study of pressure-temperature ambivalence defining the ubiquitous phase plane that characterizes both first- and second-order phase transitions. A classic example is the tricritical phase point where all three phases simultaneously co-exist; while the discontinuous jump of latent energy could drive first order phase transition, "walking around" the phase boundary could be achieved through continuous transition ${ }^{1,2}$. In most ferromagnets, the transition from the high-temperature disordered paramagnetic (PM) phase to the ferromagnetic (FM) ground state is second-order and characterized by a continuous development of magnetization below the transition point. But in some systems, FM transition often demonstrates discontinuous change in magnetization along the hysteresis path. Colossal magnetoresistive manganite is a representative example of this class of systems. In manganites $\mathrm{RE}_{1-z} \mathrm{AE}_{z} \mathrm{MnO}_{3}(\mathrm{RE}$ for rare earth ions and $\mathrm{AE}$ for alkaline earth ions), the nature of phases and transitions strongly depend on the bandwidth of the system as well as local disorder (also known as quenched disorder), arising due to the size mismatch between RE and AE cations ${ }^{3-11}$. Such disorder reduces the carrier mobility, the formation energy of lattice polarons which effectively truncates the FM phase and leads the transition towards first-order ${ }^{11}$. It has been observed that manganite with highest FMPM transition temperature $\left(T_{F M-P M}\right), \mathrm{La}_{1-z} \mathrm{Sr}_{z} \mathrm{MnO}_{3}$ with $0.2<z<0.5$ undergoes conventional second-order phase transition, whereas the lower $T_{F M-P M}$ manganites such as $\mathrm{Eu}_{1-z} \mathrm{Sr}_{z} \mathrm{MnO}_{3}$ with $0.38<z<0.47$ show evidences of strong first-order FM transition ${ }^{12,13}$. Although the order of phase transition is system dependent, it can change under the influence of various parameters. A change from first- to second-order FM transition under the influence of various external and internal perturbations is found in several theoretical ${ }^{14-19}$ and experimental works ${ }^{20-30}$. Among the manganite systems, particularly, which are very susceptible to perturbations, $\mathrm{Sm}_{1-z} \mathrm{Sr}_{z} \mathrm{MnO}_{3}$ (a narrow band manganite with relatively large disorder) is one of the prime candidate and a lot of analysis on FMPM phase transition have been done $23-27,31-38$. For $\mathrm{Sm}_{1-z} \mathrm{Sr}_{z} \mathrm{MnO}_{3}$ ( $\left.z=0.45-0.48\right)$, FM transition at ambient condition is first-order and the transition is extremely sensitive to several parameters

such as magnetic field, pressure, chemical substitution or oxygen isotope exchange, etc. ${ }^{23-27,31,34-38}$. In presence of external magnetic field $(H)$ and pressure $(P)$, the FM transition shifts towards the higher temperature while the width of thermal hysteresis in magnetization decreases gradually and eventually vanishes at a critical magnetic field-pressure phase point $\left(H_{C}, P_{C}\right)$, above which the transition becomes second-order. Similar to external pressure, the application of chemical/ internal pressure (which can be modulated by stoichiometric control) also increases the $T_{F M-P M}$ as 
observed in the partial substitution of $\mathrm{Nd}$ at $\mathrm{Sm}$-site of $\mathrm{Sm}_{1-z} \mathrm{Sr}_{z} \mathrm{MnO}_{3}\left[\left(\mathrm{Sm}_{1-x} \mathrm{Nd}_{x}\right)_{1-z} \mathrm{Sr}_{z} \mathrm{MnO}_{3}\right]$ with $z=0.45$ and 0.48 . The effect of $\mathrm{Nd}$ doping on the nature of FM transition is quite similar to that of external pressure, i.e., there exist a critical concentration $\left(x_{C}\right)$ above which the FM-PM phase boundary changes from first to second order. In other words, using parametric control of key pressure-temperature values, the first order FM transition in $\mathrm{Sm}_{1-z} \mathrm{Sr}_{z} \mathrm{MnO}_{3}$ ( $z=0.45$ and 0.48 ) could be changed over to its second ordered equivalent. Not only in ( $\mathrm{Sm}, \mathrm{Sr}) \mathrm{MnO}_{3}$, the existence of tricritical points in several other manganite systems have also been observed, which will be discussed later.

On the theoretical modeling side, few works on FM-PM phase transition of manganites have been reported ${ }^{16-19}$ but none of them characterize the nature of such phase transitions under the presence of all three external parameters - pressure, temperature and magnetic field. This is the principal focus of the present study, namely to analyze phase transition in the three dimensional phase volume. Our approach is based on a generalized version of Landau theory, integrating all three variables/ parameters. We show that both transition temperature and hysteresis width vary when these parameters change. In a particular cases, there exist tricritical points at which firstorder FM-PM transition changes to second-order. In order to illustrate the presented picture, we analyze the experimental data of our previous works ${ }^{24,26,35}$ on $\mathrm{Sm}_{0.52} \mathrm{Sr}_{0.48} \mathrm{MnO}_{3}$ single crystal, an extensively studied material showing a first-order FM transition.

\section{THEORY}

In this section, we model the critical behavior related to the FM-PM phase transition in the presence of magnetic field, pressure and chemical substitution based on Landau theory. First we discuss the effect of external magnetic field on the first-order FM phase transition.

\section{A. Effect of external magnetic field on the FM-PM phase transition}

The magnetic order is described by the magnetization $\mathbf{M}=M \hat{\mathbf{m}}$ such that $M=0$ defines the PM state and $M \neq 0$ represents the FM state. Expanding the free energy density around $M=0$, the magnetic free energy density in the presence of external $H$ can be written as

$$
F=F_{0}+\frac{1}{2} A\left(\frac{M}{M_{S}}\right)^{2}-\frac{1}{4} B\left(\frac{M}{M_{S}}\right)^{4}+\frac{1}{6} C\left(\frac{M}{M_{S}}\right)^{6}-H M
$$

where $F_{0}$ and $M_{S}$ are free energy density of PM phase and saturation magnetization, respectively. In Eq.(2.1), the coefficient $A$ can be assumed as $A=a\left(T-T^{*}\right)=a \tau$, where $a$ is a positive constant 
and $T^{*}$ is the virtual transition temperature ${ }^{2}$. The other coefficients $B$ and $C$ are assumed to be temperature-independent and positive. In the absence of magnetic field $(H=0)$, the free energy density describes a first-order FM-PM phase transition for $B>0$ while transition is second-order for $B<0$. However, in presence of external field $(H \neq 0)$, the transition may become second-order even for $B>0$. In terms of scaled magnetization $m=\frac{M}{M_{S}}$ and scaled magnetic field $h=H M_{S}$, the free energy density can be written as

$$
F=F_{0}+\frac{1}{2} A m^{2}-\frac{1}{4} B m^{4}+\frac{1}{6} C m^{6}-h m .
$$

The minimization of Eq.(2.2) gives

$$
A m-B m^{3}+C m^{5}-h=0
$$

from which one can obtain the differential equation for susceptibility (defined as $\chi=\frac{1}{\chi_{0}} \frac{\partial m}{\partial h}$ at $h=$ $0)$ as

$$
\left(A-3 B m^{2}+5 C m^{4}\right) \chi-1 / \chi_{0}=0
$$

where $m$ is the spontaneous magnetization, which can be obtained from Eq.(2.3) in the absence of external magnetic field. Eq.(2.3) infers that $m=0$ can never be a solution of $h \neq 0$ phase, which means that an induced magnetization is observed in the PM phase.

A key property of Eq. (2.3) is the change from first- to second-order FM phase transition at the critical point, characterized by $h_{C}, \tau_{C}$ and $m_{C}$, where $h_{C}, \tau_{C}$ and $m_{C}$ are the critical values of magnetic field, temperature and magnetization, respectively. The critical point is obtained from the following condition $F^{\prime}=F^{\prime \prime}=F^{\prime \prime \prime}=0$; this leads to

$$
h_{C}=H_{C} M_{S}=\frac{6 B^{2}}{25 C}\left(\frac{3 B}{10 C}\right)^{1 / 2} ; \quad \tau_{C}=T_{C}-T^{*}=\frac{9 B^{2}}{20 a C} ; \quad m_{C}=\frac{M_{C}}{M_{S}}=\left(\frac{3 B}{10 C}\right)^{1 / 2} .
$$

The complete solution of Eq. (2.3) shows the variation of $m(\tau)$ with the temperature difference $\tau=T-T^{*}$ for different $h$ values is shown in Fig. 1a, for which we have used the parameter values $a=1.0, B=3.0, C=8.0$. For $h=0, m$ drops very sharply (discontinuity in $m$ is around 0.43 ) at $\tau$ $=0.281$. With increasing field strength, the magnitude of the magnetization jump (discontinuity) starts to decrease and eventually vanishes at the critical magnetic field $h_{C} \approx 0.09$ at which zerofield FM-PM transition changes from first- to second-order. This critical line differentiating the first and second order phase transition is identified by the dotted plot.

Under the influence of $h\left(h<h_{C}\right)$, there is a shift in the first-order FM transition temperature, which can be calculated from the conditions $F-F_{0}=0$ and $\frac{\partial F}{\partial m}=0$, as

$$
T_{F M-P M}(h)=T^{*}+\frac{3 B^{2}}{16 a C}+\frac{10 h}{4 a}\left(\frac{4 C}{3 B}\right)^{1 / 2} \text {. }
$$


The evolution of magnetization $m$ [according to Eq.(2.3)] with magnetic field $h$ for different temperature values $\tau$ is shown in Fig. 1b. For $\tau_{F M-P M}(0)<\tau<\tau_{C}$, magnetization isotherms exhibit field-induced characteristic jump from low- to high-magnetic states. With increasing temperature, the sharpness of the jump decreases and no such jump exists above $\tau_{C} \approx 0.5$. The values of $h_{C}$ and $\tau_{C}$ as observed from Figures $1 \mathrm{a}$ and $1 \mathrm{~b}$ are consistent with Eq. (2.5). Here we would like to mention that the chosen values of the parameters $a, B$ and $C$ are not unique, different values of them will result different $h_{C}$ and $\tau_{C}$, but the nature of the phase diagram remains unaltered.

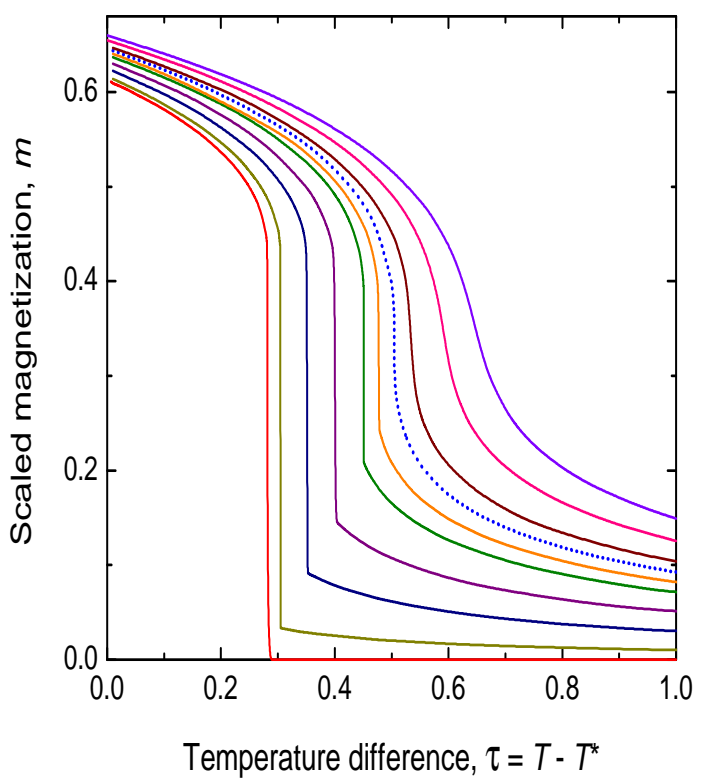

(a) Scaled magnetization, $m$ plotted against temperature difference, $\tau=T-T^{*}$ for different scaled magnetic field values $h=0$ (extreme left curve), 0.01, 0.03, 0.05, 0.07, 0.08, 0.09, 0.1, 0.12 and 0.14 (extreme right curve). The dotted line corresponds to $h=0.09 \approx h_{C}$.

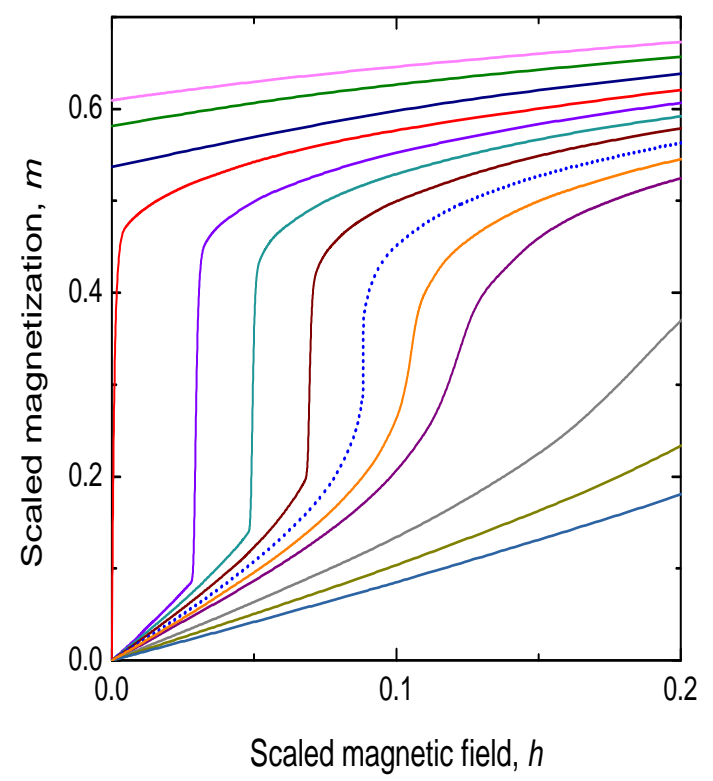

(b) $m(h)$ isotherms for different $\tau$. From top to bottom, each curve corresponds to $\tau=0.01,0.1$, $0.2,0.28,0.35,0.4,0.45,0.5,0.55,0.6,0.8,1.0$ and 1.2. The dotted line corresponds to $\tau=0.5 \approx \tau_{C}$.

FIG. 1: (Color online) Variation of scaled magnetization $m$ against scaled magnetic field $h$ and temperature difference $\tau$ from the theoretical model. All the plots have been drawn for the parameter values $a=1.0, B=3.0$ and $C=8.0$.

Now we present our previously studied experimental results analyzed on the basis of the theory discussed in the present article. The studied system was $\mathrm{Sm}_{0.52} \mathrm{Sr}_{0.48} \mathrm{MnO}_{3}$ (SSMO) single 
crystal $^{24,26}$, which was prepared by floating zone image furnace in oxygen atmosphere. The quality of the crystal was carefully checked by various techniques such as x-ray powder diffraction, Laue diffraction, electron dispersive x-ray analysis, ac susceptibility, scanning electron microscope etc. The magnetization measurements were performed in a superconducting quantum interference device magnetometer in fields up to $7 \mathrm{~T}$ using five-scan averaging.

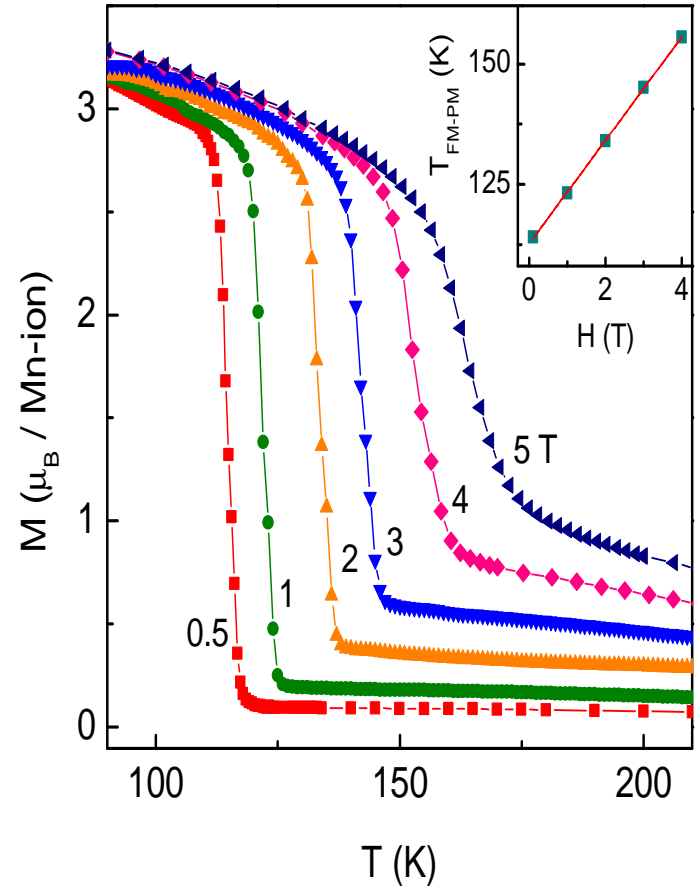

(a) $M(T)$ curves of a SSMO crystal for a range of $H$ values. The inset shows $H$-dependence of ferromagnetic-paramagnetic transition temperature $T_{\mathrm{FM}-\mathrm{PM}}$. The experimental data points (symbol) are fitted (line) from a solution of Eq. (2.6).

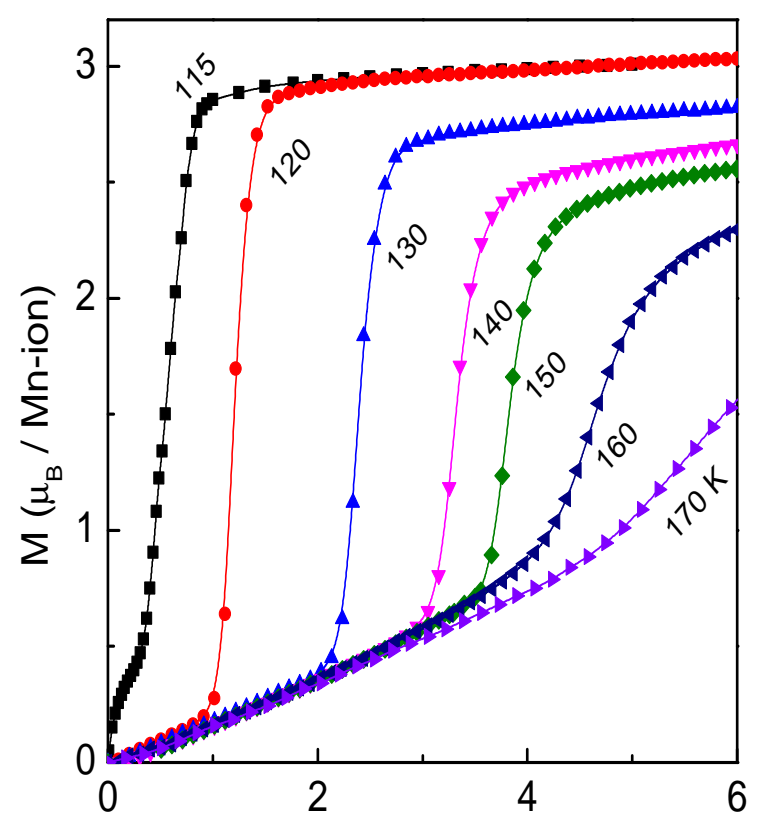

$\mathrm{H}(\mathrm{T})$

(b) $M(H)$ isotherms of an SSMO crystal demonstrating the dependence of magnetization $(M)$ on Magnetic field $(H)$ of a $\mathrm{Sm}_{0.52} \mathrm{Sr}_{0.48} \mathrm{MnO}_{3}$ (SSMO) single crystal for different temperatures $(T)$.

FIG. 2: (Color online) Variation of magnetization (M) against magnetic field $(\mathrm{H})$ and temperature (T) for a $\mathrm{Sm}_{0.52} \mathrm{Sr}_{0.48} \mathrm{MnO}_{3}$ (SSMO) single crystal.

Figure 2a shows $M(T)$ curves of SSMO crystal for a range of values of the magnetic field $H$. In the small field regime, the sharp FM-PM transition indicates that the transition is first-order in nature, which gets weakened with increasing $H$ as clearly reflected by the suppression of the magnetization change associated with the transition. Fig. 2a also depicts the nature of increase of the FM-PM transition temperature with increasing external field; here we have fitted the data 
displayed in this figure using Eq. (2.6), specifically using the functional forms $\left(T^{*}+\frac{3 B^{2}}{16 a C}\right)=$

$112.7 \pm 0.2 \mathrm{~K}$ and $\left(\frac{10 M_{S}}{4 a}\right) \sqrt{\frac{4 C}{3 B}}=10.74 \pm 0.08 \mathrm{~K} / \mathrm{T}$ as fitting parameters. In a related system $\mathrm{Sm}_{0.55} \mathrm{Sr}_{0.45} \mathrm{MnO}_{3}{ }^{25}$, where $\mathrm{Sr}$ concentration differs slightly, FM to PM phase transition is firstorder with $T_{F M-P M}(H=0) \sim 135 \mathrm{~K}$. With the application of external field, the transition is shifted to higher temperatures at an average rate of $\mathrm{d} T_{F M-P M} / \mathrm{d} H=9 \mathrm{~K} / \mathrm{T}$. Simultaneously, the first-order nature of the transition weakens and above a critical point $\left(H_{C} \approx 3.75 \mathrm{~T}, T_{C} \approx 165.4\right.$ $\mathrm{K})$, the transition becomes second-order. The field-induced change in the character of the FM-PM transition is observed in other manganites also such as $\mathrm{Sm}_{0.55}\left(\mathrm{Sr}_{0.5} \mathrm{Ca}_{0.5}\right)_{0.45} \mathrm{MnO}_{3}\left(H_{C} \approx 11.5 \mathrm{~T}\right.$, $\left.T_{C} \approx 164 \mathrm{~K}\right)^{28}, \mathrm{Eu}_{0.55} \mathrm{Sr}_{0.45} \mathrm{MnO}_{3}\left(H_{C} \approx 7.4 \mathrm{~T}\right)^{25}$ and $\mathrm{La}_{1-x} \mathrm{Ca}_{x} \mathrm{MnO}_{3}$ with $x=0.25\left(H_{C} \approx 4\right.$ $\mathrm{T})^{22}$ and $0.3\left(H_{C} \approx 6.5 \mathrm{~T}\right)^{30}$.

The evolution of $M$ with $H$ for different temperatures is shown in Fig. 2b. It is clear that $M(H)$ isotherms are not conventional Brillouin-like, rather they exhibit field-induced metamagnetic transition. With increasing $T$, the sharpness of the jump decreases. From a comparison with observations detailed in the next section, it is clear that the temperature and field dependence of magnetization observed in SSMO are qualitatively similar to the magnetization behavior, calculated from free energy density [Eq.(2.3)], as shown in Figures 1a and 1b. For SSMO, the experimentally observed critical point at which zero-field first-order FM transition becomes secondorder is given by $H_{C} \approx 4 T ; \quad M_{C} \approx 0.88 \mu_{B} /$ Mn-ion; $T_{C} \approx 160 \mathrm{~K}$.

\section{B. Effect of external pressure on the FM-PM phase transition}

To study the pressure dependent change in FM-PM transition, we consider a strong coupling between magnetic order parameter and lattice strain. So the free energy density in the presence of external pressure can be written as

$$
\begin{aligned}
F= & F_{0}+\frac{1}{2} A\left(\frac{M}{M_{S}}\right)^{2}-\frac{1}{4} B\left(\frac{M}{M_{S}}\right)^{4}+\frac{1}{6} C\left(\frac{M}{M_{S}}\right)^{6}+\frac{1}{2} u_{i j k l} \epsilon_{i j} \epsilon_{j k} \\
& -\frac{1}{2} \eta_{1} \epsilon_{i i}\left(\frac{M}{M_{S}}\right)^{2}+\frac{1}{2} \delta_{1} \epsilon_{i i}^{2}\left(\frac{M}{M_{S}}\right)^{2}-P_{i j} \epsilon_{i j}
\end{aligned}
$$

The cross-coupling terms $\eta_{1}$ and $\delta_{1}$ are assumed to be positive and they characterize the coupling strength between the magnetic order parameter and strain tensor $\epsilon$. The positive signs of $\eta_{1}$ and $\delta_{1}$ ensure the increase of magnetization and FM transition temperature with the increase of pressure [see Eq. (2.12)]. The last term of Eq. (2.7) represents the coupling between pressure and elastic 
strain. In terms of scaled magnetization $m, F$ can be rewritten as

$$
F=F_{0}+\frac{1}{2} A m^{2}-\frac{1}{4} B m^{4}+\frac{1}{6} C m^{6}+\frac{1}{2} u \epsilon^{2}-\frac{1}{2} \eta_{1} \epsilon m^{2}+\frac{1}{2} \delta_{1} \epsilon^{2} m^{2}-P \epsilon,
$$

Now the minimization of Eq. (2.8) with respect to $\epsilon$ we get

$$
\epsilon=\frac{P+\eta^{*} m^{2}+\delta^{*} m^{4}}{u}
$$

with $\eta^{*}=\frac{1}{2} \eta_{1}-\frac{\delta_{1}}{u} P$ and $\delta^{*}=-\frac{\delta_{1} \eta_{1}}{2 u}$. Equation (2.9) shows that strain changes with temperature and pressure since $m$ changes with temperature. Elimination of $\epsilon$ in Eq. (2.8) yields

$$
F=F_{0}^{*}+\frac{1}{2} A^{*} m^{2}-\frac{1}{4} B^{*} m^{4}+\frac{1}{6} C^{*} m^{6}
$$

where $F_{0}^{*}=F_{0}-\frac{P^{2}}{2 u}$ and the renormalized coefficients are given by

$$
A^{*}=A-\frac{\eta_{1} P}{u}+O\left(P^{2}\right), \quad B^{*}=B+\frac{\eta_{1}^{2}}{2 u}-\frac{2 \delta_{1} \eta_{1}}{u} P+O\left(P^{2}\right), \quad C^{*}=C+\frac{5 \delta_{1} \eta_{1}^{2}}{4 u^{2}}-\frac{5 \delta_{1}^{2} \eta_{1}}{2 u^{3}} P+O\left(P^{2}\right) .
$$

The value of the magnetization in the ferromagnetic state can be calculated after the minimization of Eq. (2.10) and can be expressed as

$$
m^{2}=\frac{B^{*}}{2 C^{*}}\left[1+\left[1-\frac{4 C^{*}}{B^{* 2}}\left(a\left(T-T^{*}\right)-\frac{\eta_{1} P}{u}\right)\right]^{1 / 2}\right],
$$

Eq. (2.12) shows that the magnetization increases with increase of pressure which agrees with experiments $^{26,27}$. In the FM phase, $T-T^{*}$ is negative and hence the coupling constant $\eta_{1}$ should be positive to ensure the increase of magnetization with increase of external pressure. Moreover, the form of the free energy density as shown in Eq. (2.10) clearly shows that the jump of the order parameter $m_{F M-P M}=\left(3 B^{*} / 4 C^{*}\right)^{1 / 2}$ decreases with increase of pressure. To show more clearly the variation of the magnetization with temperature as well as pressure in the FM phase, we have plotted $m^{2}$ as a function of temperature for different pressure (Fig. 3). This is done for a set of phenomenological parameters for which the FM-PM transition is possible. From Fig. 3, one can see that with increasing pressure, both magnetization and FM transition temperature increase, whereas the jump of the order parameter at the transition point diminishes, indicating the closeness of second order character of the FM-PM transition.

The pressure dependent susceptibility can be calculated by adding a term $-H M$ in the free energy expansion Eq. (2.10) and then the differential equation for susceptibility can be written as

$$
\left(A^{*}-3 B^{*} m^{2}-5 C^{*} m^{4}\right) \chi-1 / \chi_{0}=0,
$$

where the magnetization $m$ can be obtained from Eq. (2.12) in the absence of the external magnetic field. 


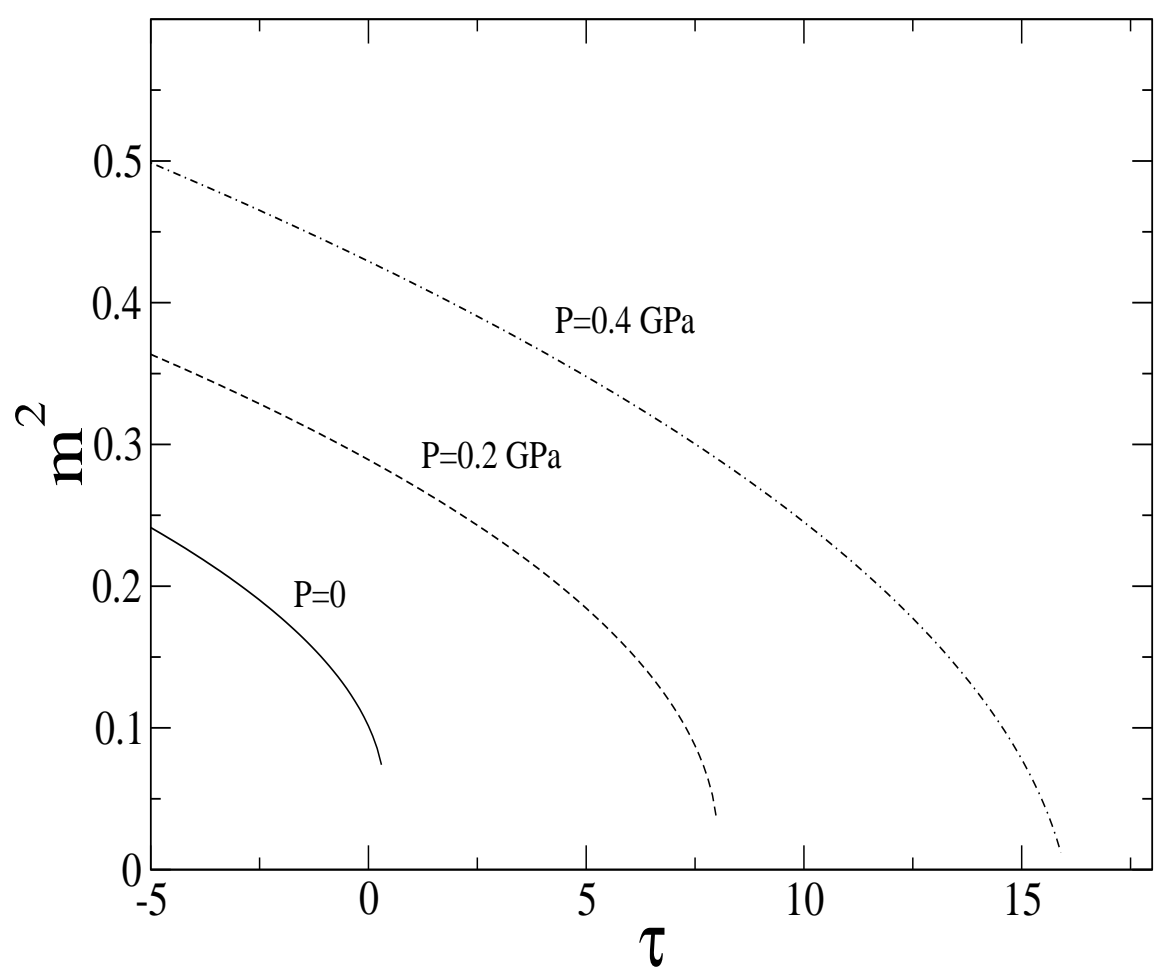

FIG. 3: (Color online) $m^{2}$ vs. $\tau$ for different pressure. The plots have been evaluated for the parameter values $a=0.1, B=0.5, C=0.8, \eta_{1}=2.0, \delta_{1}=0.7$ and $u=0.5$.

The pressure dependent first order FM-PM phase transition can be calculated from the condition $F-F_{0}=0$ and $\frac{\partial F}{\partial m}=0$ as

$$
T_{F M-P M}(P)=T^{*}+\frac{\eta_{1} P}{a u}+\frac{3 B^{* 2}}{16 a C^{*}}
$$

After simplification, Eq. (2.14) can be rewritten as

$$
T_{F M-P M}(P)=T^{*}+\frac{w}{16 a C}+v_{1} P+O\left(P^{2}\right)
$$

where $w=\left(B+\frac{\eta_{1}^{2}}{2 u}\right)^{2}\left(1-\frac{5 \delta_{1} \eta_{1}^{2}}{4 u^{2} C}\right)$ and $v_{1}=\frac{\eta_{1}}{a u}+\frac{5 \delta_{1}^{2} \eta_{1}}{2 u^{3} C}\left(1+\frac{\eta_{1}^{2}}{2 u B}\right)^{2}-\frac{4 \delta_{1} \eta_{1}}{u}\left(1-\frac{5 \delta_{1} \eta_{1}^{2}}{4 u^{2} C}\right)\left(1+\frac{\eta_{1}^{2}}{2 u B}\right)$.

The spread of thermal hysteresis around the FM-PM phase transition point can be calculated from the condition $F^{\prime}=F^{\prime \prime}=0$ and is expressed as

$$
\triangle T_{F M-P M}(P)=T^{* *}-T_{1}^{*}=\frac{B^{* 2}}{4 a C^{*}}
$$


where the supercooling temperature $T_{1}^{*}=T^{*}+\frac{\eta_{1} P}{a u} . T^{* *}$ is the temperature of the superheated ferromagnetic phase. Equation (2.16) can be rewritten as

$$
\triangle T_{F M-P M}(P)=\frac{w_{1}}{4 a C}+v_{2} P
$$

where $w_{1}=B^{2}\left(1-\frac{5 \delta_{1} \eta_{1}^{2}}{4 u^{2} C}\right)$ and $v_{2}=-\frac{5 \delta_{1}^{2} \eta_{1}}{32 u^{3} C}\left(B+\frac{\eta_{1}^{2}}{2 u}\right)^{2}+\frac{\delta_{1} \eta_{1}}{4 u}\left(1-\frac{5 \delta_{1} \eta_{1}^{2}}{4 u^{2} C}\right)\left(B+\frac{\eta_{1}^{2}}{2 u}\right)$.

From Eq. (2.11), it is clear that as pressure changes the renormalized coefficients $A^{*}$ and $B^{*}$ change and hence order of the FM transition also changes. For weak coupling and the lower value of pressure, $B^{*}>0$, i.e., $B>\frac{2 \delta_{1} \eta_{1}}{u} P-\frac{\eta_{1}^{2}}{2 u}$, indicating that the FM-PM phase transition is first order, where both FM and PM phases coexist, i.e., a thermal hysteresis appears [see Eq. (2.16)]. As pressure increases, $B^{*}$ starts to decrease and for strong coupling and high value of $P, B^{*}<0$ i.e. $B<\frac{2 \delta_{1} \eta_{1}}{u} P-\frac{\eta_{1}^{2}}{2 u}$, then a second order transition occurs. Thus at critical pressure $P_{C}$, thermal hysteresis vanishes and the first-order FM-PM transition becomes second-order in nature. For a particular value of the pressure, $B^{*}=0$, then the first-order FM transition crosses over to the second order transition i.e. a tricritical point is obtained. Hence, a tricritical point can be achieved by varying external pressure $P$ only. The quantitative nature of the pressure/doping dependence on magnetization explained through Eqs. (2.13-2.17) are structurally reminiscent of Eqs. (2.3-2.6), and hence will show qualitative similarity with Figs. 1 and 2 discussed earlier.

In order to justify our proposed theory, we present our previous experimental work on SSMO single crystal $^{23,26}$. The effect of external pressure (up to $2 \mathrm{GPa}$ ) on the nature of FM to PM phase transition has been studied. With increasing pressure, $T_{F M-P M}$ increases while the width of thermal hysteresis reduces as shown in Fig. (4). We fit these experimentally measured data points according to Eqs. (2.15) and (2.17) which clearly points to the strong agreement of our theoretical model based results with real experimental data. For this, we have used the fit parameters $\left(T^{*}+\frac{w}{16 a C}\right)$ $=110.4 \pm 0.7 \mathrm{~K}, v_{1}=17.8 \pm 0.5 \mathrm{~K} / \mathrm{GPa}, \frac{w_{1}}{4 a C}=4.39 \pm 0.04 \mathrm{~K}$ and $v_{2}=-1.62 \pm 0.03 \mathrm{~K} / \mathrm{GPa}$. For SSMO, the value of critical pressure where the zero-field transition becomes second-order is $P_{C} \approx 2.7 \mathrm{GPa}$. In $\mathrm{Sm}_{0.55} \mathrm{Sr}_{0.45} \mathrm{MnO}_{3}{ }^{25}$, the application of pressure increases $T_{F M-P M}$ linearly at the rate of $\sim 20 \mathrm{~K} / \mathrm{GPa}$, while $\Delta T_{F M-P M}$ narrows down. The critical pressure where the transition changes its character is estimated to be $P_{C} \approx 3.2 \mathrm{GPa}$. 


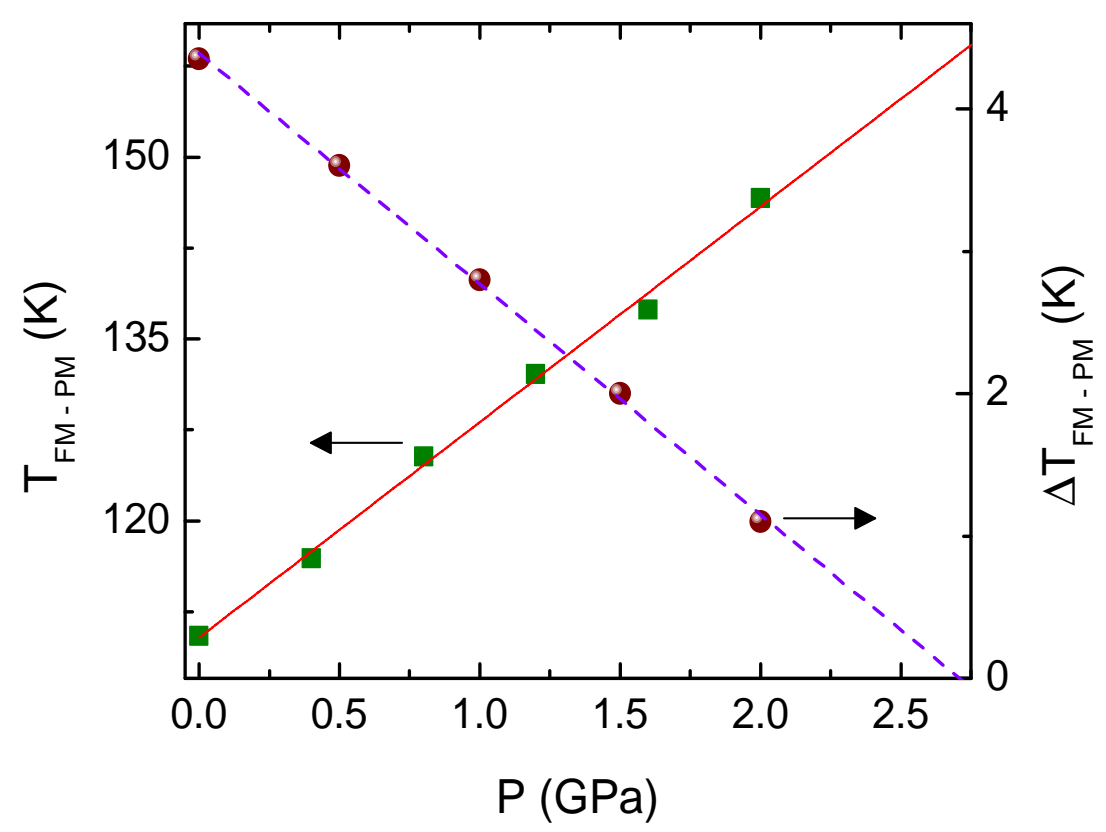

FIG. 4: (Color online) Pressure $(P)$ dependence of FM transition temperature $\left(T_{F M-P M}\right)$ and thermal hysteresis width $\left(\Delta T_{F M-P M}\right)$ of SSMO single crystal. The symbols are experimentally measured data points and the solid and dashed lines are the best fit of Eqs. (2.15) and (2.17), respectively.

\section{Effect of chemical substitution on the FM-PM phase transition}

Let us now consider the effect of chemical substitution on the FM-PM phase transition. In the case of binary mixture of impurity, the free energy must be expressed in terms of the order parameter and impurity concentration $x$. The simplest way to take into account the effect of impurity is to introduce the impurity-magnetization coupling terms in the free energy expression which becomes

$$
F=F_{0}+\frac{1}{2} A\left(\frac{M}{M_{S}}\right)^{2}-\frac{1}{4} B\left(\frac{M}{M_{S}}\right)^{4}+\frac{1}{6} C\left(\frac{M}{M_{S}}\right)^{6}-\frac{1}{2} D\left(\frac{M}{M_{S}}\right)^{2} x+\frac{1}{2} E\left(\frac{M}{M_{S}}\right)^{2} x^{2}+\frac{1}{2} G x^{2} .
$$

where $A=a\left(T-T^{*}\right)$. The term $\frac{1}{2} G x^{2}$ is the free energy density of the impurity solute. $D$ and $E$ are the coupling constants. All the coefficients $B, C, D, G$ and $E$ are assumed to be positive to ensure the increase of transition temperature [see Eq.(2.23)] with $x$. Since the order of FM to PM phase transition depends on the sign of the coefficient $B$. We assume $B$ changes with concentration and we set $B=b_{0}\left(x-x_{0}\right)$, where $x_{0}$ is the equilibrium value of concentration and $b_{0}$ is a positive 
constant $^{39}$.

Taking the partial derivate of Eq. (2.18) with respect to $x$, we get

$$
\frac{\partial F}{\partial x}=G x-\frac{1}{2} D m^{2}+E m^{2} x \equiv \mu
$$

where $m=\frac{M}{M_{S}}$ and $\mu$ is the quantity thermodynamically conjugate to $x$. Simplifying Eq. (2.19), we get

$$
x=\frac{1}{G}\left[\mu\left(1-\frac{E m^{2}}{G}\right)+\frac{1}{2} D m^{2}-\frac{1}{2} \frac{E D}{G} m^{4}\right] .
$$

Applying Legendre transformation, we have

$$
\begin{aligned}
F(m, \mu, T)= & F(m, x, T)-\mu x \\
& =F_{0}^{* *}+\frac{1}{2} A^{* *} m^{2}-\frac{1}{4} B^{* *} m^{4}+\frac{1}{6} C^{* *} m^{6},
\end{aligned}
$$

where $F_{0}^{* *}=F_{0}-\frac{\mu^{2}}{2 G}$ and the renormalized coefficients are given by

$$
A^{* *}=A-\frac{\mu D}{G}+\frac{\mu^{2} E}{G^{2}} ; \quad B^{* *}=B+\frac{7 D^{2}}{2 G}-\frac{\mu E D}{G^{2}}+\frac{\mu^{2} E^{2}}{G^{3}} ; \quad C^{* *}=C+\frac{21 E D^{2}}{4 G^{2}}+\frac{3 \mu^{2} E^{3}}{G^{4}} .
$$

It is clear from Eq. (2.22) that the consideration of couplings between magnetization $M$ and impurity concentration $x$ leads to the renormalization of the coefficients $A, B$ and $C$. The coefficient $B$ changes with $x$, which means that the order of FM-PM transition can also change with impurity concentration. For weak coupling constants $E$ and $D$ and low value of $x, B^{* *}>0$ and $C^{* *}>0$, i.e., FM-PM transition is first-order in nature. Then the concentration dependence of FM-PM transition temperature can be calculated following the procedure as in Eq. (2.14) to get

$$
T_{F M-P M}(x)=T^{*}+\frac{3 B^{2}}{16 a C}+\frac{D x}{a}-\frac{E x^{2}}{a} .
$$

Similar to the procedure adapted in Eq. (2.16), the width of thermal hysteresis is given by

$$
\triangle T_{F M-P M}(x)=\frac{3 B^{2}}{4 a C}
$$

Equation (2.23) shows that first order FM-PM transition temperatures increases with the increase of concentration. This shows that the coupling constants $E$ and $D$ should be positive.

Now for strong coupling constants $E$ and $D$ and higher value of concentration $x, B^{* *}<0$, the transition is second order. For a particular value of the concentration $x=x_{t c p}, B^{* *}=0$, the first order transition goes to a second order transition. So, there is a crossover from first to second order transition and a tricritical point appears. 
From the condition $A^{* *}=0$, the second order transition can be expressed as

$$
T_{C}=T^{*}+\frac{D x}{a}-\frac{E x^{2}}{a}
$$

In our previous experimental study ${ }^{26}$, we have observed the doping dependence change in the order of FM-PM transition. The studied system was $\left(\mathrm{Sm}_{1-x} \mathrm{Nd}_{x}\right)_{0.52} \mathrm{Sr}_{0.48} \mathrm{MnO}_{3}$ single crystals with $0 \leq x \leq 0.3$. The magnetization data of parent compound $\operatorname{SSMO}(x=0)$ shows a firstorder FM to $\mathrm{PM}$ phase transition at $T_{F M-P M} \approx 114 \mathrm{~K}$ along with thermal hysteresis of width $\triangle T_{F M-P M} \approx 4.3 \mathrm{~K}$. With the substitution of Nd at Sm site, $T_{F M-P M}$ increases while $\triangle T_{F M-P M}$ decreases, which are displayed in Fig. 5. These experimental data (solid symbols) are fitted to Eqs. (2.23) and (2.24). The best-fit parameters are given by $T^{*}=109.3 \pm 0.4 \mathrm{~K}, \frac{B_{0}^{2}}{16 a C}=3.54 \pm$ $0.07 \mathrm{~K}, x_{0}=0.64 \pm 0.03, D / a=129.7 \pm 0.6 \mathrm{~K}, E / a=38.1 \pm 0.6 \mathrm{~K}$; the corresponding curves (solid lines) are as shown in Fig. 5. The complete phase diagrams of $\left(\mathrm{Sm}_{1-x} \mathrm{Nd}_{x}\right)_{0.55} \mathrm{Sr}_{0.45} \mathrm{MnO}_{3}$ single crystals have been studied for $0 \leq x \leq 0.5$ by Demkó et al. ${ }^{25}$. For $x=0$, FM transition is first-order with $T_{F M-P M} \approx 135 \mathrm{~K}$ and $\triangle T_{F M-P M} \approx 1.7 \mathrm{~K}$. With increasing Nd concentration, $T_{F M-P M}$ increases and $\triangle T_{F M-P M}$ narrows down. Above a critical concentration $x_{C} \approx 0.33$, the hysteresis completely vanishes and the first-order transition becomes second-order. Similar kind of behavior has also observed in $\mathrm{La}_{1-z} \mathrm{Ca}_{z} \mathrm{MnO}_{3}$, where first-order $(z<0.4) \mathrm{FM}$ to PM phase transition changes to second-order $(z>0.4)$ with increasing Ca doping ${ }^{20}$.

\section{CONCLUSIONS}

We have discussed the FM-PM phase transition based on a phenomenological Ginzburg-Landau theory. In the presence of magnetic field, first order FM-PM phase transition can become second ordered at the critical point. The effect of pressure on the FM-PM phase transition is to increase the transition temperature. The theory predicts a second order character of the FM-PM phase transition at high pressure. The formation of two phase regions during the FM-PM phase transition, prompted by chemical substitution, depends on the small values of the coefficients $B$ and $C$. The different values of the Landau coefficients indicate the change of the transition temperatures and the second order character of the transition. In a mixture, the FM-PM phase transition which is first order of the pure form becomes a second ordered transition with the change of concentration. This leads to a crossover from first to second order transition via a tricritical point. This study 


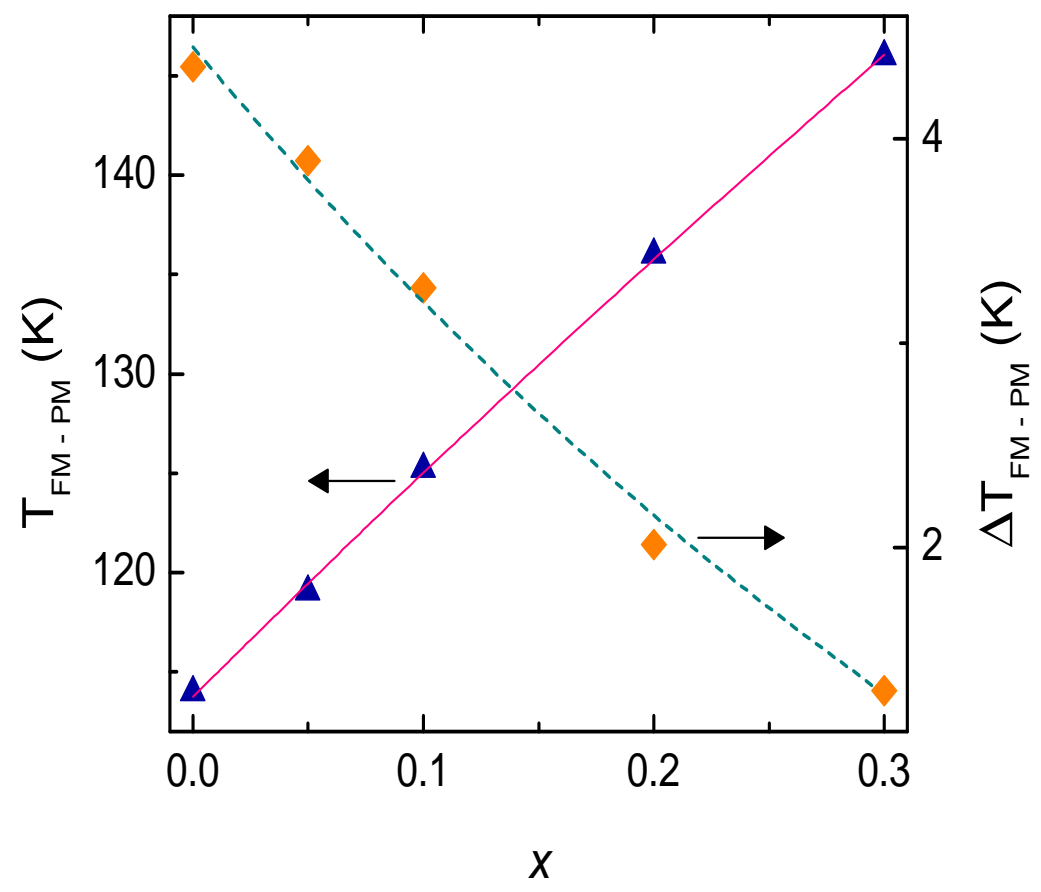

FIG. 5: (Color online) Ferromagnetic-paramagnetic transition temperature $\left(T_{F M-P M}\right)$ and thermal hysteresis width $\left(\Delta T_{F M-P M}\right)$ of $\left(\mathrm{Sm}_{1-x} \mathrm{Nd}_{x}\right)_{0.52} \mathrm{Sr}_{0.48} \mathrm{MnO}_{3}$ single crystals as a function of Nd concentration $(x)$. The symbols represent experimental data points while the solid and dotted lines are the best fit of Eqs. (2.23) and (2.24), respectively.

thus presents a theory based confirmation of experimental results both at the qualitative and quantitative levels.

As shown from comparison with other experimental results on manganites like $\mathrm{Sm}_{0.55}\left(\mathrm{Sr}_{0.5} \mathrm{Ca}_{0.5}\right)_{0.45} \mathrm{MnO}_{3}$, $\mathrm{Sm}_{0.55} \mathrm{Sr}_{0.45} \mathrm{MnO}_{3}, \mathrm{Sm}_{0.52} \mathrm{Sr}_{0.48} \mathrm{MnO}_{3}, \mathrm{Sm}_{0.55}\left(\mathrm{Sr}_{0.5} \mathrm{Ca}_{0.5}\right)_{0.45} \mathrm{MnO}_{3}, \mathrm{Eu}_{0.55} \mathrm{Sr}_{0.45} \mathrm{MnO}_{3}$ and $\mathrm{La}_{1-x} \mathrm{Ca}_{x} \mathrm{MnO}_{3}$, the variation of FM-PM transition temperature and hysteresis width with external/ internal perturbations conform to our model results. Changes in the character of FM phase transition under the influence of external magnetic field, pressure and chemical substitution observed in several manganite systems ${ }^{20,22-29,35,36}$ have all been satisfactorily explained within the remit of our theoretical model justifying the occurrence of a tricritical point, a hitherto unexplained feature in most studies. 


\section{ACKNOWLEDGMENT}

PS is sincerely grateful to Professor P Mandal for his many valuable suggestions and for a critical reading of the manuscript. AKC acknowledges partial financial support to SARI, Aston.

* pkmuk1966@gmail.com

$\dagger$ psphysics1981@gmail.com

$\ddagger$ a.k.chattopadhyay@aston.ac.uk

1 H. E. Stanley, Introduction to Phase Transitions and Critical Phenomena, (Oxford University Press, New York, 1971).

2 S. K. Ma, Modern Theory of Critical Phenomena, (Addison-Wesley, 1976).

3 C.N.R. Rao, B. Raveau, Colossal Magnetoresistance, Charge Ordering and Related Properties of Manganese Oxides, (World Scientific, Singapore, 1998).

4 E. Dagotto, T. Hotta, and A. Moreo, Phys. Rep. 344, 1 (2001).

5 E. Dagotto, Science 309, 257 (2005).

6 Y. Tokura, Rep. Prog. Phys. 69, 797 (2006).

7 H. Y. Hwang, S. W. Cheong, P. G. Radaelli, M. Marezio, and B. Batlogg, Phys. Rev. Lett. 75, 914 (1995).

8 L. M. Rodriguez-Martinez and J. P. Attfield, Phys. Rev. B 54, R15622 (1996); 58, 2426 (1998); 63, $024424(2000)$.

9 R. Mahesh and M. Itoh, Phys. Rev. B 60, 2994 (1999).

10 Y. Tomioka and Y. Tokura, Phys. Rev. B 70, 14432 (2004).

11 T. J. Sato, J. W. Lynn, and B. Dabrowski, Phys. Rev. Lett. 93, 267204 (2004).

12 K. Ghosh, C. J. Lobb, R. L. Greene, S. G. Karabashev, D. A. Shulyatev, A. A. Arsenov, and Y. Mukovskii, Phys. Rev. Lett. 81, 4740 (1998).

13 Y. Tomioka, R. Kumai, T. Ito, and Y. Tokura, Phys. Rev. B 80, 174414 (2009).

14 Y. Imry and M. Wortis, Phys. Rev. B 19, 3580 (1979).

15 A. N. Das and B. Ghosh, J. Phys. C 16, 1799 (1983).

16 A. S. Alexandrov and A.M. Bratkovsky, Phys. Rev. Lett. 82, 141 (1999).

17 J. A. Verges, V. Martin-Mayor, and L. Brey, Phys. Rev. Lett. 88, 136401 (2002).

18 S. W. Biernacki, Phys. Rev. B 68, 174417 (2003).

19 S. Bustingorry, F. Pomiro, G. Aurelio, and J. Curiale, Phys. Rev. B 93, 224429 (2016).

20 D. Kim, B. Revaz, B. L. Zink, F. Hellman, J. J. Rhyne, and J. F. Mitchell, Phys. Rev. Lett. 89, 227202 $(2002)$. 
21 V. S. Amaral, J. P. Araujo, Yu. G. Pogorelov, J. B. Sousa, P. B. Tavares, J. M. Vieira, P. A. Algarabel, and M. R. Ibarra, J. Appl. Phys. 93, 7646 (2003).

22 B. I. Belevtsev, G. A. Zvyagina, K. R. Zhekov, I. G. Kolobov, E. Yu. Beliayev, A. S. Panfilov, N. N. Galtsov, A. I. Prokhvatilov, and J. Fink-Finowicki, Phys. Rev. B 74, 054427 (2006).

23 K. Mydeen, P. Sarkar, P. Mandal, A. Murugeswari, C. Q. Jin, and S. Arumugam, Appl. Phys. Lett. 92, 182510 (2008).

24 P. Sarkar, P. Mandal, A. K. Bera, S. M. Yusuf, L. S. SharathChandra, and V. Ganesan, Phys. Rev. B 78, 012415 (2008).

25 L. Demkó, I. Kézsmárki, G. Mihály, N. Takeshita, Y. Tomioka, and Y. Tokura, Phys. Rev. Lett. 101, 037206 (2008).

26 P. Sarkar, P. Mandal, K. Mydeen, A. K. Bera, S. M. Yusuf, S. Arumugam, C. Q. Jin, T. Ishida, and S. Noguchi, Phys. Rev. B 79, 144431 (2009).

27 P. Sarkar, S. Arumugam, P. Mandal, A. Murugeswari, R. Thiyagarajan, S. EsakiMuthu, D. MohanRadheep, C. Ganguli, K. Matsubayshi, and Y. Uwatoko, Phys. Rev. Lett. 103, 057205 (2009).

28 D. M. Radheep, P. Sarkar, S. Arumugam, R. Suryanarayanan, and P. Mandal, J. Magn. Magn. Mater. 365, 51 (2014).

29 N. Khan, P. Sarkar, A. Midya, P. Mandal, and P. K. Mohanty, Sci. Rep. 7, 45004 (2017).

30 T. L. Phan, P. S. Tola, N. T. Dang, J. S. Rhyee, W. H. Shon, T. A. Ho, J. Magn. Magn. Mater. 441, 290 (2017).

31 N. A. Babushkina, E. A. Chistotina, O. Yu. Gorbenko, A. R. Kaul, D. I. Khomskii, and K. I. Kugel, Phys. Rev. B 67, 100410(R) (2003).

32 L. M. Fisher, A. V. Kalinov, I. F. Voloshin, N. A. Babushkina, D. I. Khomskii, Y. Zhang, and T. T. M. Palstra, Phys. Rev. B 70, 212411 (2004).

33 Y. Tomioka, H. Hiraka, Y. Endoh, and Y. Tokura, Phys. Rev. B 74, 104420 (2006).

34 G. S. Chang, E. Z. Kurmaev, L. D. Finkelstein, N. A. Babushkina, A. Moewes, and T. A. Callcott, Phys. Rev. B 74, 125105 (2006).

35 P. Sarkar and P. Mandal, Appl. Phys. Lett. 92, 052501 (2008).

36 P. Sarkar, P. Mandal, and P. Choudhury, Appl. Phys. Lett. 92, 182506 (2008).

37 A. Murugeswari, P. Sarkar, S. Arumugam, N. Manivannan, P. Mandal, T. Ishida, and S. Noguchi, Appl. Phys. Lett. 94, 252506 (2009).

38 M. M. Saber, M. Egilmez, A. I. Mansour, I. Fan, K. H. Chow, and J. Jung, Phys. Rev. B 82, 172401 (2010).

39 P. K. Mukherjee, RSC Advances 5, 12168 (2015). 